\title{
Efficacy of the interpersonal and social
} rhythm therapy (IPSRT) in patients with bipolar disorder: results from a real-world, controlled trial

\author{
Luca Steardo $\mathrm{Jr}^{1,2^{*}}$, Mario Luciano ${ }^{1}$, Gaia Sampogna ${ }^{1}$, Francesca Zinno ${ }^{1}$, Pasquale Saviano ${ }^{3}$, Filippo Staltari ${ }^{2}$, \\ Cristina Segura Garcia ${ }^{4}$, Pasquale De Fazio ${ }^{2}$ and Andrea Fiorillo ${ }^{1}$
}

\begin{abstract}
Background: Bipolar disorder (BD) is one of the most burdensome mental disorder, with a lifetime prevalence of $2.4 \%$, with a prevalence of $0.6 \%$ for bipolar type I and $0.4 \%$ for bipolar type II. Several interventions have been developed to implement the treatment strategy of bipolar disorder, including the Interpersonal and Social Rhythm Therapy (IPSRT). This intervention has been specifically developed to manage patients'stressful life events, improve the disruptions of social and circadian rhythms and increase adherence to medications. The aim of the present study is to assess the efficacy of IPSRT on affective and anxiety psychopathology, social functioning, response to pharmacological treatment and affective morbidity index (AMI) in BD patients.
\end{abstract}

Methods: BD patients were consecutively recruited at the Mood Disorder Unit of the University of Campania "Luigi Vanvitelli" and randomly assigned to the experimental group receiving the IPSRT or to the Treatment as Usual (TAU) group. Patients were assessed at baseline, after 3 and 6 months with several validated assessment tools and with the affective morbidity index.

Results: At the end of the intervention, compared to controls, patients from the experimental group reported a significant improvement in anxious depressive and manic symptomatology, global functioning; and response to mood stabilizers. Patients in the IPSRT group reported a reduction at the AMI score.

Conclusions: IPSRT has been confirmed to be effective in improving the clinical symptomology of BD patients and in improving the affective morbidity index. Further studies with longer follow-up are needed in order to assess the stability of the results.

Trial registration The study was approved by the local ethical review board (N001567/28.01.2018)

Keywords: Bipolar disorder, Psychosocial intervention, Affective morbidity index, Treatment strategy

*Correspondence: lucasteardo@gmail.com

1 Department of Psychiatry, University of Campania "Luigi Vanvitelli", Largo

Madonna Delle Grazie, 80138 Naples, Italy

Full list of author information is available at the end of the article

\section{Introduction}

Bipolar disorder is one of the most burdensome mental disorder, with a lifetime prevalence of $2.4 \%$, and a prevalence of $0.6 \%$ for bipolar I and $0.4 \%$ for bipolar II subtypes [1]. It poses a significant burden on patients, their relatives and the society at large. It is characterized by mood swings in both polarities, manic and depressive, 
and heterogeneous symptoms, involving affective, cognitive and physical alterations. Its management includes pharmacological agents such as mood stabilizers, second-generation antipsychotics, antidepressants as well as psychosocial interventions, including cognitive behavioral therapy (CBT) [2, 3], psychoeducation (PE) [4-8], family-focused therapy (FFT) and interpersonal and social rhythm therapy (IPSRT) [9-11]. Although pharmacotherapy is the recommended first-line therapy for manic, depressive and residual states, medication adherence is typically poor, relapse rates are high, and full remission is not always achieved. Psychosocial therapies have been found to be effective in terms of improvement of medication adherence, identification of early warning signs, increasing in self-management skills and in family communication, all elements which could potentially explain the reduced rate of relapses in patients treated with these interventions. Therefore, the integration between pharmacological and psychosocial interventions is essential for the optimal management of patients with bipolar disorders and should be implemented in the clinical routine care [12]. In fact, several international guidelines [13-16] recommend their use as adjunctive interventions to pharmacological treatment for the long-term management of bipolar disorder in both the acute and maintenance phases $[17,18]$. These different approaches share several aims, including: (1) the improvement of sleep disturbances; (2) the promotion of healthy lifestyle behaviors; (3) the monitoring of mood shifts; (4) the early recognition of patients' warning signs; (5) the improvement of problem-oriented coping strategies [19].

IPSRT has been specifically developed to manage patients' stressful life events, improve the disruptions of social and circadian rhythms and increase their adherence to medications [20]. The IPSRT is based on the theoretical approaches of the interpersonal psychotherapy (IPT) and social rhythm therapy. The efficacy of IPSRT on the outcome of BD patients has been tested only in a handful of studies [21-25]. Frank et al. [20] found that IPSRT is associated with a better outcome in terms of remission from manic symptoms in a cohort of $44 \mathrm{BD}$ patients, compared to patients receiving pharmacotherapy only. Furthermore, in a RCT carried out by the same authors [26], the IPSRT was associated with a longer euthymic period compared to patients from the control group.

In this study, we evaluated the efficacy of IPSRT in terms of reduction of levels of patients' affective and anxiety psychopathology, social functioning, response to pharmacological treatment and affective morbidity index (AMI), compared to a group of patients receiving standard care.

\section{Methods}

\section{Participants}

The study has been conducted at the Mood Disorder Unit of the University of Campania "Luigi Vanvitelli" in Naples (Italy) during the period January 2018 to February 2019.

Patients were included in the study if they met the following criteria: (1) age between 18 and 70 years; (2) a DSM-5 diagnosis of type-I or type-II bipolar disorder, confirmed through Structured Clinical Interview for DSM-5 disorders, clinician version-SCID-5-CV [27]; (3) stable treatment with mood stabilizers (at least 1 year duration and, in the case of lithium or valproic acid, at therapeutic blood levels); (4) willingness to participate in the study, expressed by written informed consent provided upon complete description of the protocol. Patients were excluded in case of: (1) inability to give a written consent to participate in the study; (2) diagnosis of any neurologic disease; (3) presence of drug and/or alcohol abuse; (4) receiving any other psychotherapeutic intervention at the moment of the recruitment.

Patients were consecutively recruited and randomly assigned to the experimental group receiving the IPSRT or to the control group, receiving the pharmacotherapy treatment plus treatment as usual.

The study has been carried out in accordance with the latest version of the Declaration of Helsinki. All patients gave their written informed consent and were enrolled in the study.

The study was approved by the local ethical review board (N001567/28.01.2018).

\section{Procedures}

Patients' socio-demographic (i.e., gender, age at study entry, employment status and level of education) and clinical characteristics (i.e., age at onset, age at first psychiatric contact, duration of illness, lifetime number of affective episodes, pattern of illness course, presence of mixed affective states, and number of suicidal attempts) were recorded with an ad hoc schedule. Clinical course and treatment history for each patient were evaluated through the National Institute of Mental Health Life Chart Method at baseline [18]. Patients have been assessed at baseline (T0), and after 3 (T1) and 6 months (T2).

\section{Psychopathological assessments}

All recruited patients were assessed through the administration of: (a) the Inventory of Depressive Symptomatology (Self-Report) (IDS-SR) [28]; (b) the Montgomery Asberg Depression Rating scale (MADRS) [29]; (c) the 14-item Hamilton Rating Scale for Anxiety (HAM-A) [30]; (d) the Mania Rating Scale (MRS) [31]; (e) the global assessment of functioning (GAF) [32]. Two psychometric 
tools have been adopted for assessing depressive symptoms, in order to obtain an objective assessment of depressive symptomatology as well as the personal perception of patients, through a self-report scale.

Patients' response to mood stabilizers was assessed by the Retrospective Criteria of Long-term Treatment Response in Bipolar Disorder (the ALDA Scale), which consists of two criteria: (a) association between clinical improvement and treatment; and (b) degree of the causal relationship between clinical improvement and prophylactic treatment. A total score was obtained by subtracting the (b) from the (a) score [33, 34].

The clinical course of BD was assessed with the Affective Morbidity Index (AMI; $[35,36]$ ), which considers the duration and the severity of mood episodes. Symptoms' severity is rated as follows: (1) symptoms not requiring any treatment modification; (2) symptoms requiring an adjunctive pharmacological intervention, but not a dosage change of mood stabilizers; (3) symptoms requiring treatment modification to be managed within hospitalization. AMI absolute value was calculated according to the following formula: $\mathrm{AMI}=$ (weeks with degree 1) + (weeks with degree 2$) \times 2+$ (weeks with degree $3) \times 3$ total number of weeks $\times 3$.

The AMI was calculated for the first 3 months (AMIT1), from the third until the sixth month (AMI-T2) and during the whole treatment period $\left(\mathrm{AMI}_{\text {tot }}\right)$. The variation of the AMI index was considered a measure of psychological improvement.

\section{The interpersonal and social rhythm therapy}

IPSRT is a psychosocial intervention specifically developed to address circadian dysregulation in BD and it is based on the principles of social rhythm therapy, interpersonal psychotherapy and psychoeducation [20]. The intervention focuses on the four main areas of interpersonal psychotherapy (grief, role of transition, role of disputes and interpersonal deficits) combined with strategies to improve the social and circadian rhythms. In addition, psychoeducational sessions are provided to improve patients' compliance to pharmacological treatments. It consists of 12 weekly sessions, each lasting about $90 \mathrm{~min}$. All participating patients were asked to fill in the social rhythm metric scale.

The intervention is divided in four phases: (1) the initial two sessions are focused on illness history and aim to identify the relationship between stressful life events and mood shifts; (2) the second phase includes four sessions focused on the reorganization of social rhythms and the increase of skills to cope with social stressors; (3) the maintenance phase (four sessions) focuses on reinforcing new social rhythms and building confidence in learned techniques in order to prevent future affective episodes;
(4) the final phase consists of two sessions, during which the skills achieved with the IPSRT are further discussed and advices for the future are given.

All patients received individual sessions of IPSRT. The intervention was provided by a trained psychiatrist (LS).

\section{Statistical analyses}

Descriptive statistics included frequencies and means, as appropriate. Differences between the two groups were explored through Chi-squared and $t$-tests, as appropriate. The General Linear Model (GLM)-Repeated Measures test was used to explore variations of HAM-A, MRS, IDSSR, MADRS and GAF in both groups in the 6-month period $\left(T_{0}, T_{1}\right.$ and $\left.T_{2}\right)$. Eta-squared, a measure of effect size in ANOVA, was calculated for significant results. The AMI was calculated as the sum of the weeks of degree 1 , weeks with degree two multiplied for two, weeks with degree three multiplied for three divided for the total number of weeks multiplied for three. The level of statistical significance was set at $p<0.05$. Data analyses have been made with the Statistical Package for Social Sciences Version 21 (SPSS, Chicago, Illinois, USA).

\section{Results}

The final sample consists of $44 \mathrm{BD}$ patients, who were randomly allocated to the experimental or to the control group. All patients allocated in the IPSRT group attended all sessions of the intervention, with $100 \%$ retention rate.

The main socio-demographic and clinical characteristics of recruited patients are reported in Table 1. The two samples did not differ with regard to socio-demographic and clinical variables, with the only exception of diagnosis distribution and clinical course. In the experimental group, 8 patients were treated with valproate $(900 \mathrm{mg} /$ day), one with lithium (900 mg/day), 8 with lithium in combination with antipsychotics and 5 with valproate in combination with other medications. In the control group, 5 patients were treated with valproate $(900 \mathrm{mg} /$ day), 10 with lithium (900 mg/day), 7 with lithium in combination with antipsychotics. Differences between the two groups emerged about the diagnosis $\left(\chi^{2}=5,350\right.$; $d f=1 p=0.021)$ and clinical course $\left(\chi^{2}=14,186 ; d f=3\right.$; $p=0.003)$; no statistical differences have been found for all other considered variables.

Data on the efficacy of the IPSRT on AMI index are reported in Table 2 and in Fig. 1; the results of GLM repeated measures are reported in Table 2. At the end of the intervention, compared to controls, patients from the experimental group reported a significant improvement in anxious symptoms (HAM-A: $p<0.001 ; \eta^{2}=0.389$ ), manic symptoms (MRS: $p<0.004 ; \quad \eta^{2}=0.234$ ), depressive symptoms (IDSSR: $p<0.007 ; \quad \eta^{2}=0.216$; MADRS: $p<0.057$ ), global functioning (GAF: $p<0.001$; 
Table 1 Socio-demographic and clinical characteristics of the global sample

\begin{tabular}{|c|c|c|c|c|c|c|c|}
\hline \multirow[b]{2}{*}{ Sex (male, \%) } & \multicolumn{2}{|c|}{ IPSRT group } & \multicolumn{2}{|c|}{ TAU group } & \multirow{2}{*}{$\begin{array}{l}\text { Group comparison } \\
0.093\end{array}$} & \multirow{2}{*}{$\frac{d f}{1}$} & \multirow{2}{*}{$\begin{array}{l}p \\
0.761\end{array}$} \\
\hline & 10 & 46 & 9 & 41 & & & \\
\hline Age $(M \pm S D)^{a}$ & 49.1 & 12.6 & 46.7 & 12.8 & $t=0.629$ & 42 & 0.533 \\
\hline \multicolumn{8}{|l|}{ Civil status ${ }^{b}$} \\
\hline Single & 14 & 64 & 10 & 46 & $x^{2}=2.889$ & 2 & 0.236 \\
\hline Married & 8 & 36 & 10 & 46 & & & \\
\hline Divorced & 0 & 0 & 2 & 9 & & & \\
\hline Education (years) ${ }^{a}$ & 13.9 & 3.7 & 13.6 & 3,2 & $t=0.263$ & 42 & 0.794 \\
\hline Employed (yes) ${ }^{b}$ & 11 & 50 & 14 & 64.6 & $x^{2}=0.834$ & 1 & 0.361 \\
\hline \multicolumn{8}{|l|}{ Diagnosis ${ }^{\mathrm{b}}$} \\
\hline $\mathrm{BD} \mid$ & 19 & 86 & 12 & 55 & $x^{2}=5.350$ & 1 & 0.021 \\
\hline $\mathrm{BD} \|$ & 2 & 14 & 10 & 46 & & & \\
\hline Family psychiatric history (yes) ${ }^{b}$ & 15 & 68 & 14 & 64 & $x^{2}=0.101$ & 1 & 0.750 \\
\hline Family history for other disorders (yes) & 7 & 32 & 11 & 50 & $x^{2}=1.504$ & 1 & 0.220 \\
\hline Comorbidity (yes) ${ }^{\mathrm{b}}$ & 8 & 36 & 12 & 55 & $x^{2}=1.467$ & 1 & 0.226 \\
\hline Age at onset ${ }^{a}$ & 28.5 & 8.8 & 28.8 & 14.8 & $t=-0.099$ & 42 & 0.921 \\
\hline Age at first psychiatric visit ${ }^{a}$ & 28.5 & 8.7 & 32 & 13.9 & $t=-0.987$ & 42 & 0.329 \\
\hline Age at first hospitalization ${ }^{\mathrm{a}}$ & 33.1 & 13.2 & 28 & 8.8 & $t=0.944$ & 17 & 0.358 \\
\hline Age at first depressive episode ${ }^{a}$ & 29 & 9.8 & 28.8 & 13.5 & $t=0.056$ & 40 & 0.955 \\
\hline Age at first manic episode ${ }^{a}$ & 32 & 13.3 & 28.1 & 7.9 & $t=0.921$ & 29 & 0.365 \\
\hline Age first hypomanic episode ${ }^{a}$ & 31.1 & 9.4 & 34.1 & 14.3 & $t=-0.623$ & 25 & 0.539 \\
\hline \multicolumn{8}{|l|}{ Course $^{b}$} \\
\hline $\mathrm{MDI}$ & 12 & 55 & 11 & 50 & $x^{2}=14.186$ & 3 & 0.003 \\
\hline MID & 3 & 14 & 4 & 18 & & & \\
\hline DMl & 7 & 32 & 0 & 0 & & & \\
\hline $\mathrm{IRR}$ & 0 & 0 & 7 & 32 & & & \\
\hline Number of depressive episodes ${ }^{\mathrm{a}}$ & 4.1 & 3.6 & 3.7 & 3.5 & $t=0.345$ & 40 & 0.732 \\
\hline Number of manic episodes ${ }^{a}$ & 2.5 & 2.1 & 2.9 & 2.6 & $t=-0.552$ & 32 & 0.585 \\
\hline Number of hypomanic episodes ${ }^{a}$ & 1,7 & 2,3 & 2.8 & 2.2 & $t=-1.564$ & 36 & 0.127 \\
\hline Suicide attempts (yes) ${ }^{\mathrm{b}}$ & 2 & 9 & 2 & 9 & $x^{2}=0$ & 1 & 1.000 \\
\hline Psychotic symptoms (yes) ${ }^{b}$ & 12 & 55 & 9 & 41 & $x^{2}=0.820$ & 1 & 0.365 \\
\hline Aggressiveness (yes) ${ }^{b}$ & 15 & 68 & 13 & 59 & $x^{2}=0.393$ & 1 & 0.531 \\
\hline \multicolumn{8}{|l|}{ Number depressive episode last year ${ }^{b}$} \\
\hline 0 & 5 & 23 & 6 & 27 & $x^{2}=1.091$ & 2 & 0.580 \\
\hline 1 & 15 & 68 & 12 & 55 & & & \\
\hline 2 & 2 & 9 & 4 & 18 & & & \\
\hline
\end{tabular}

BD / bipolar disorder type I, BD // bipolar disorder type II, MDI manic-depression interval, MID manic-interval depression, DMI depression-manic interval, IRR irregular

a Data are expressed as mean and standard deviations

b Data are expressed as frequencies and percentages

$\eta^{2}=0.309$ ); and response to mood stabilizers (ALDA scale: $p<0.001 ; \eta^{2}=0.300$ ). Moreover, patients receiving the experimental intervention also had an improvement in psychological functioning, as confirmed by the reduction in the AMI score $\left(p<0.001 ; \eta^{2}=0.341\right)$.

\section{Discussion}

One of the main findings of our study is the improvement of manic, anxiety and depressive symptoms in patients receiving the IPSRT compared to controls [37].
The reduction of psychopathological burden remained stable after 6 months in the experimental group. The intervention was well-received by patients and was associated with a high retention rate, as highlighted by the fact that none of the recruited patients dropped out [38, 39]. Moreover, the IPSRT can be easily implemented in the routine care of mental health centers considering that it can be administered by all categories of mental health professionals (including psychiatrists, psychologists, nurses, social workers and psychiatric rehabilitation 
Table 2 Differences at total scores' scales between the two groups at baseline, after 3 and 6 months

\begin{tabular}{|c|c|c|c|c|c|c|c|}
\hline & Group & $T_{0}$ & $T_{1}$ & $T_{2}$ & $F$ & $p$ & $\eta^{2 a}$ \\
\hline \multirow[t]{2}{*}{ HAM-A } & Group 1 & 8.636 & 1.773 & 0.545 & Time: $F(2,41)=0.816$ & .449 & \\
\hline & Group 2 & 5.773 & 13.455 & 17.773 & Group: $F(2,41)=13.076$ & $<.001$ & 0.389 \\
\hline \multirow[t]{2}{*}{ MRS } & Group 1 & 9.864 & 0.636 & 0.727 & Time: $F(2,41)=2.428$ & .101 & \\
\hline & Group 2 & 5.500 & 7.273 & 8.091 & Group: $F(2,41)=6.275$ & .004 & 0.234 \\
\hline \multirow[t]{2}{*}{ IDSSR } & Group 1 & 16.273 & 11.318 & 3.727 & Time: $F(2,41)=1.099$ & .343 & \\
\hline & Group 2 & 15.136 & 28.955 & 25.273 & Group: $F(2,41)=5.655$ & .007 & 0.216 \\
\hline \multirow[t]{2}{*}{ MADRS } & Group 1 & 7.636 & 4.182 & 1.545 & Time: $F(2,41)=0.796$ & .458 & \\
\hline & Group 2 & 6.364 & 8.955 & 8.318 & Group: $F(2,41)=3.073$ & .057 & \\
\hline \multirow[t]{2}{*}{ GAF } & Group 1 & 67.636 & 78.091 & 78.500 & Time: $F(2,41)=1.293$ & .285 & \\
\hline & Group 2 & 73.864 & 68.909 & 69.227 & Group: $F(2,41)=9.170$ & .001 & 0.309 \\
\hline \multirow[t]{2}{*}{ ALDA scale } & Group 1 & 4.818 & 5.091 & 5.273 & Time: $F(2,41)=1.187$ & .315 & \\
\hline & Group 2 & 4.864 & 4.273 & 4.227 & Group: $F(2,41)=8.792$ & .001 & 0.300 \\
\hline \multirow[t]{2}{*}{ AMl } & Group 1 & & 0.547 & 0.437 & Time: $F(2,41)=21.700$ & $<.001$ & 0.341 \\
\hline & Group 2 & & 0.713 & 0.713 & Group: $F(2,41)=21.700$ & $<.001$ & 0.341 \\
\hline
\end{tabular}

Group 1: control group, Group 2: IPSRT group

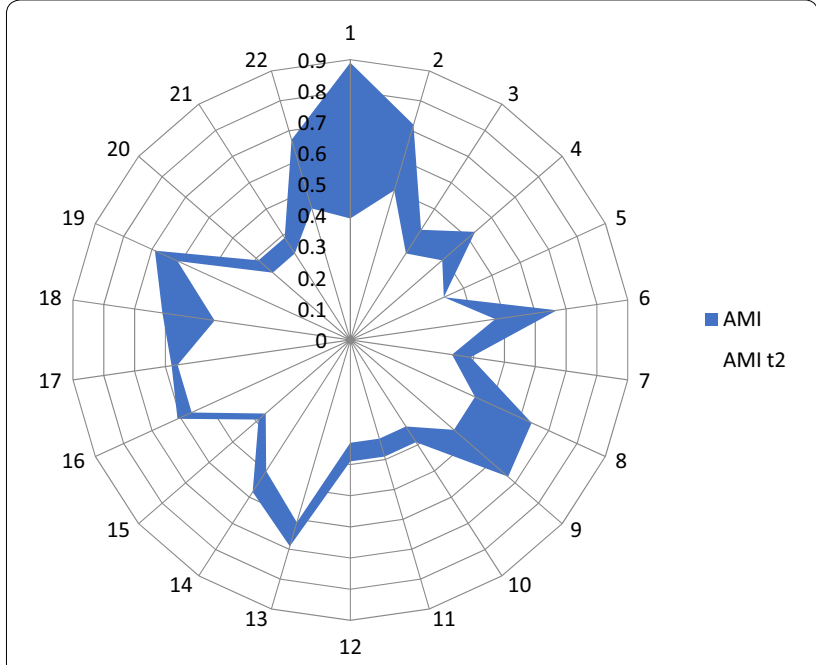

Fig. 1 Reduction of AMI index in patients' experimental group

technicians) after a brief training [40, 41]. The positive effect of the intervention on all symptom domains can be explained by several factors, including that the strong relationship between the improvement of circadian rhythms and symptomatic remission in patients with BDs [42-44]. In fact, sleep dysregulation is considered a trigger factor for the development of manic or depressive symptoms $[45,46]$. The IPSRT has a specific focus on sleep disturbances through the monitoring of daily levels of energy, thus contributing to a better daily planning. Another possible explanation for the improvement of the levels of psychiatric symptoms is the fact that our intervention included sessions on the improvement of patients' compliance to psychopharmacological medications $[47,48]$. In particular, information on possible side effects of medications are regularly provided to patients and their concerns about medications and side effects are regularly addressed, during the treatment period.

Another relevant finding of our study is the improvement of anxiety symptoms. Anxiety is very common in $\mathrm{BD}$ patient and is associated with poor treatment response [49]. The IPSRT contributes to reduce the levels of anxiety by helping patients to address their interpersonal deficits and improving their emotional dysregulation, and not just by managing affective symptoms. Similar results have been reported with other psychotherapeutic approaches, such as cognitive behavioral therapy (CBT) and mindfulness-based cognitive therapy (MCBT) [50]. As expected, at the follow-up we observed an improvement of GAF score. This result emphasizes the importance of the interpersonal intervention in improving all aspects of patients' life, thus contributing to prevent mood shifts [51].

The differences between the two groups at the Alda scale total score can be due to the improvement of patients' adherence to medications following their participation at the IPSRT.

In our study, the provision of IPSRT significantly improved the AMI index. To the best of our knowledge, this is the first study which tested the efficacy of a psychosocial intervention in BD through AMI. This instrument gives an accurate picture of psychopathological status as it assesses the severity and length of mood shifts over the lifetime [52].

Our results confirm the importance of complementing the pharmacological treatment with psychotherapy 
in bipolar disorder in a naturalistic setting $[7,8]$. Our patients treated with IPSRT plus pharmacotherapy reported an improvement of affective and anxiety symptoms without requiring any dose adjustment of mood stabilizers. This result, confirmed by the reduction of AMI index at 3 and 6 months, emphasizes the importance of IPSRT in reducing the psychological burden of bipolar disorder and preventing mood relapses [53, 54].

This study has some limitations that need to be acknowledged. In particular, the sample size was small and the follow-up was short. In fact, to establish a strong association between IPSRT and mood improvement, longer follow-ups (i.e., at 12 and 18 months) with larger samples are needed. Moreover, the adoption of the MRS for evaluating manic symptoms should limit the generalizability and external validity of our findings. However, this is a not funded study conducted in the clinical routine care and we decide to use an assessment instrument routinely used in the bipolar unit of the mental health department, in order to reduce the burden for participating professionals.

Furthermore, patients' satisfaction with the received treatment has not been measured through ad hoc validated instrument. Our study can be considered as a pilot and we are planning a follow-up study with the same cohort of patients in order to verify if our encouraging results at 6 months are confirmed and for evaluating patients' expectations and levels of satisfaction with the received treatment.

\section{Conclusions}

The main finding of the present study is the reduction of the psychopathological burden in BD patients treated with IPSRT, as shown by the reduction of total score scale after 6 months and changing in affective morbidity index. Moreover, this intervention has been well received by patients and it is feasible in clinical naturalistic setting. Further studies should be conducted in order to evaluate in long-term efficacy in terms on reduction of relapses.

\section{Acknowledgements \\ The authors are grateful to all patients participating in the study.}

\section{Authors' contributions}

LS, ML, and PDF wrote the first draft of the paper. CSG and LS performed the statistical analyses. GS, AF and PS contributed to the second and final version of the paper. FZ and FS conducted the references'search. All authors read and approved the final manuscript.

\section{Funding}

No funding was received.

\section{Availability of data and materials}

All data generated or analyzed during this study are included in this published article.

\section{Ethics approval and consent to participate}

The study has been carried out in accordance with the latest version of the Declaration of Helsinki. All patients gave their written informed consent and were enrolled in the study.

\section{Consent for publication}

Not applicable.

\section{Competing interests}

The authors declare that they have no competing interests.

\section{Author details}

1 Department of Psychiatry, University of Campania "Luigi Vanvitelli", Largo Madonna Delle Grazie, 80138 Naples, Italy. ${ }^{2}$ Psychiatric Unit, Department of Health Sciences, University Magna Graecia, Catanzaro, Italy. ${ }^{3}$ UOSM Nola, DSM ASL NA 3 Sud, Nola, Italy. ${ }^{4}$ Department of Medical and Surgical Sciences, University Magna Graecia of Catanzaro, Catanzaro, Italy.

Received: 28 January 2020 Accepted: 21 February 2020

Published online: 09 March 2020

\section{References}

1. Merikangas KR, Jin R, He JP, et al. Prevalence and correlates of bipolar spectrum disorder in the world mental health survey initiative. Arch Gen Psychiatry. 2011;68:241-51.

2. Cuijpers P. Targets and outcomes of psychotherapies for mental disorders: an overview. World Psychiatry. 2019;18:276-85.

3. Lilienfeld SO. What is "evidence" in psychotherapies? World Psychiatry. 2019;18:245-6.

4. Reinares M, Sanchez-Moreno J, Fountoulakis KN. Psychosocial interventions in bipolar disorder: what, for whom, and when. J Affect Disord. 2014;156:4655.

5. Luciano M, Del Vecchio V, Sampogna G, De Rosa C, Fiorillo A. Including family members in psychoeducation for bipolar disorder: is it worth it? Bipolar Disord. 2015;17:458-9.

6. Luciano M, Sampogna G, Del Vecchio V, Giacco D, Mulè A, De Rosa C, et al. The family in Italy: cultural changes and implications for treatment. Int Rev Psychiatry. 2012;24:149-56.

7. Fiorillo A, Del Vecchio V, Luciano M, Sampogna G, De Rosa C, Malangone C, et al. Efficacy of psychoeducational family intervention for bipolar I disorder: A controlled, multicentric, real-world study. J Affect Disord. 2015;172:291-9.

8. Fiorillo A, Del Vecchio V, Luciano M, Sampogna G, Sbordone D, Catapano F, et al. Feasibility of a psychoeducational family intervention for people with bipolar I disorder and their relatives: Results from an Italian realworld multicentre study. J Affect Disord. 2016;190:657-62.

9. Depp CA, Ceglowski J, Wang VC, Yaghouti F, Mausbach BT, Thompson WK, et al. Augmenting psychoeducation with a mobile intervention for bipolar disorder: a randomized controlled trial. J Affect Disord. 2015;174:23-30.

10. Cardoso T, Mondin TC, Reyes AN, Zeni CP, Souza LD, da Silva RA, et al. Biological rhythm and bipolar disorder: twelve-month follow-up of a randomized clinical trial. J Nerv Ment Dis. 2015;203:792-7.

11. Smith DJ, Griffiths E, Poole R, di Florio A, Barnes E, Kelly MJ, et al. Beating bipolar: exploratory trial of a novel Internet-based psychoeducational treatment for bipolar disorder. Bipolar Disord. 2011;13:571-7.

12. Chatterton ML, Stockings E, Berk M, Barendregt JJ, Carter R, Mihalopoulos C. Psychosocial therapies for the adjunctive treatment of bipolar disorder in adults: network meta-analysis. Br J Psychiatry. 2017;210:333-41.

13. Yatham LN, Kennedy SH, Parikh SV, Schaffer A, Bond DJ, Frey BN. Canadian Network for Mood and Anxiety Treatments (CANMAT) and International Society for Bipolar Disorders (ISBD) 2018 guidelines for the management of patients with bipolar disorder. Bipolar Disord. 2018;20:97-170.

14. Konstantinos N, Yatham LF, Grunze H, Vieta E, Young A, Blier P, et al. The International College of Neuro-Psychopharmacology (CINP) Treatment Guidelines for Bipolar Disorder in Adults (CINP-BD-2017), part 2: Review, grading of the evidence, and a precise algorithm. Int J Neuropsychopharmacol. 2017;20:121-79. 
15. Kendall T, Morriss R, Mayo-Wilson E, Meyer TD, Jones SH, Oud M, et al. NICE guidance on psychological treatments for bipolar disorder. Lancet Psychiatry. 2016:3:317-20.

16. Grunze H, Vieta E, Goodwin GM, Bowden C, Licht RW, Möller HJ, et al. WFSBP Task Force on Treatment Guidelines for Bipolar Disorders. The World Federation of Societies of Biological Psychiatry (WFSBP) Guidelines for the biological treatment of bipolar disorders: update 2012 on the long-term treatment of bipolar disorder. World J Biol Psychiatry. 2013;14:154-21919.

17. Parikh SV, Hawke LD, Velyvis V, Zaretsky A, Beaulieu S, Patelis-Siotis I, et al. Combined treatment: impact of optimal psychotherapy and medication in bipolar disorder. Bipolar Disord. 2015;17:86-96.

18. Sampogna G, Luciano M, Del Vecchio V, Malangone C, De Rosa C, Giallonardo $V$, et al. The effects of psychoeducational family intervention on coping strategies of relatives of patients with bipolar I disorder: results from a controlled, real-world, multicentric study. Neuropsychiatr Dis Treat. 2018;14:977-89.

19. Salcedo S, Gold AK, Sheikh S, Marcus PH, Nierenberg AA, Deckersbach T, et al. Empirically supported psychosocial interventions for bipolar disorder: current state of the research. J Affect Disord. 2016;201:20314.

20. Frank E, Hlastala S, Ritenour A, Houck P, Tu XM, Monk TH, et al. Inducing lifestyle regularity in recovering bipolar disorder patients: results from the maintenance therapies in bipolar disorder protocol. Biol Psychiatry. 1997:41:1165-73.

21. Hlastala SA, Kotler JS, McClellan JM, McCauley EA. Interpersonal and social rhythm therapy for adolescents with bipolar disorder: treatment development and results from an open trial. Depress Anxiety. 2010;27:457-64.

22. Goldstein TR, Fersch-Podrat R, Axelson DA, Gilbert A, Hlastala SA, Birmaher $B$, Frank E. Early intervention for adolescents at high risk for the development of bipolar disorder: pilot study of Interpersonal and Social Rhythm Therapy (IPSRT). Psychotherapy. 2014;51:180-9.

23. Haynes PL, Gengler D, Kelly M. Social Rhythm Therapies for Mood Disorders: an Update. Curr Psychiatry Rep. 2016;18:75.

24. Hoberg AA, Ponto J, Nelson PJ, Frye MA. Group interpersonal and social rhythm therapy for bipolar depression. Perspect Psychiatr Care. 2013;49:226-34.

25. Inder ML, Crowe MT, Luty SE, Carter JD, Moor S, Frampton CM, et al. Randomized, controlled trial of Interpersonal and Social Rhythm Therapy for young people with bipolar disorder. Bipolar Disord. 2015;17:128-38.

26. Frank E, Kupfer DJ, Thase ME, Mallinger AG, Swartz HA, Fagiolini A, et al. Two-year outcomes for interpersonal and social rhythm therapy in individuals with bipolar I disorder. Arch Gen Psychiatry. 2005;62:996-1004.

27. First MB, Williams JB, Karg RS, Spitzer RL. SCID-5-CV: structured clinical interview for DSM-5 disorders, clinician version. Arlington: American Psychiatric Association; 2015.

28. Rush AJ, Gullion CM, Basco MR, Jarrett RB, Trivedi MH. The inventory of depressive symptomatology (IDS): psychometric properties. Psychol Med. 1996;26:477-86.

29. Montgomery SA, Asberg M. A new depression scale designed to be sensitive to change. Br J Psychiatry. 1979;134:382-9.

30. Maier W, Buller R, Philipp M, Heuser I. The Hamilton Anxiety Scale: reliability, validity and sensitivity to change in anxiety and depressive disorders. J Affect Disord. 1988;14:61-8.

31. Bech P, Rafaelsen OJ, Kramp P, Bolwig TG. The Mania Rating Scale: Scale construction and inter-observer agreement. Neuropharmacology. 1978;17:430-1.

32. Jones SH, Thornicroft G, Coffey M, Dunn G. A brief mental health outcome scale-reliability and validity of the Global Assessment of Functioning (GAF). Br J Psychiatry. 1995;166:654-9.

33. Steardo L Jr, Fabrazzo M, Sampogna G, Monteleone AM, D'Agostino G, Monteleone $\mathrm{P}$, et al. Impaired glucose metabolism in bipolar patients and response to mood stabilizer treatments. J Affect Disord. 2019;245:174-9.

34. Manchia M, Adli M, Akula N, Ardau R, Aubry JM, Backlund L, et al. Assessment of response to lithium maintenance treatment in bipolar disorder: Consortium on Lithium Genetics (ConLiGen) report. PLoS ONE. 2013;8:e65636.

35. Cairns K, McCarvill T, Ruzickova M, Calkin CV. Course of bipolar illness worsens after onset of insulin resistance. J Psychiatr Res. 2018;102:34-7.

36. Denicoff KD, Leverich GS, Nolen WA, Rush AJ, McElroy SL, Keck PE, et al. Validation of the prospective NIMH-Life-Chart Method (NIMH-LCM-p) for longitudinal assessment of bipolar illness. Psychol Med. 2000;30:1391-7.

37. van Ommeren M. Targets and outcomes of psychological interventions: implications for quidelines and policy. World Psychiatry. 2019:18:295-6.

38. Clarke E, Puschner B, Jordan H, Williams P, Konrad J, KawohI W, et al. Empowerment and satisfaction in a multinational study of routine clinical practice. Acta Psychiatr Scand. 2015;131:369-78.

39. Leichsenring F, Sarrar L, Steinert C. Drop-outs in psychotherapy: a change of perspective. World Psychiatry. 2019;18:32-3.

40. Priebe S, Miglietta E. Assessment and determinants of patient satisfaction with mental health care. World Psychiatry. 2019;18:30-1.

41. Pingani L, Fiorillo A, Luciano M, Catellani S, Vinci V, Ferrari S, et al. Who cares for it? How to provide psychosocial interventions in the community. Int J Soc Psychiatry. 2013;59:701-5.

42. Fiorillo A, Luciano M, Pompili M, Sartorius N. Editorial: reducing the mortality gap in people with severe mental disorders: the role of lifestyle psychosocial interventions. Front Psychiatry. 2019;10:434.

43. Sampogna G, Fiorillo A, Luciano M, Del Vecchio V, Steardo L Jr, Pocai B, et al. A randomized controlled trial on the efficacy of a psychosocial behavioral intervention to improve the lifestyle of patients with severe mental disorders: study protocol. Front Psychiatry. 2018;9:235.

44. Geoffrey P, Boudebesse C, Bellivier F, Lajnef M, Henry C, Leboyer M, et al. Sleep in remitted bipolar disorder: a naturalistic case-control study using actigraphy. J Affect Disord. 2014;158:1-7.

45. De Rosa C, Sampogna G, Luciano M, Del Vecchio V, Pocai B, Borriello G, et al. Improving physical health of patients with severe mental disorders: a critical review of lifestyle psychosocial interventions. Expert Rev Neurother. 2017;17:667-81.

46. Friess E, Modell S, Brunner H, Tagaya H, Lauer CJ. The Munich vulnerability study on affective disorders: microstructure of sleep in high-risk subjects. Eur Arch Psychiatry Clin Neurosci. 2008;258:285-91.

47. García S, Martínez-Cengotitabengoa M, López-Zurbano S, et al. Adherence to antipsychotic medication in bipolar disorder and schizophrenic patients: a systematic review. J Clin Psychopharmacol. 2016;36:355-71.

48. Giacco D, Luciano M, Del Vecchio V, Sampogna G, Slade M, Clarke E, et al. Desire for information of people with severe mental illness. Soc Psychiatry Psychiatr Epidemiol. 2014;49:1883-911.

49. Deckersbach T, Peters AT, Sylvia L, Urdahl A, Magalhães PV, Otto MW, et al. Do comorbid anxiety disorders moderate the effects of psychotherapy for bipolar disorder? Results from STEP-BD. Am J Psychiatry. 2014;171:178-86.

50. Wolkenstein L, Zwick JC, Hautzinger M, Joormann J. Cognitive emotion regulation in euthymic bipolar disorder. J Affect Disord. 2014;160:92-7.

51. Alloy LB, Nusslock R. Reward-related cognitive vulnerability to bipolar spectrum disorders. World Psychiatry. 2018;17:102-3.

52. International Consortium on Lithium Genetics (ConLi+Gen). Association of Polygenic Score for Schizophrenia and HLA antigen and inflammation genes with response to lithium in bipolar affective disorder: a genomewide association study. JAMA Psychiatry. 2018;75:65-74.

53. Gross JJ, Uusberg H, Uusberg A. Mental illness and well-being: an affect regulation perspective. World Psychiatry. 2019;18:130-9.

54. Masten AS. Resilience from a developmental systems perspective. World Psychiatry. 2019;18:101-2.

\section{Publisher's Note}

Springer Nature remains neutral with regard to jurisdictional claims in published maps and institutional affiliations. 